\title{
Normal and abnormal lipid metabolism in children
}

\author{
BARRY LEWIS \\ M.D., Ph.D., M.R.C.Path., F.R.C.P. \\ Chemical Pathology and Metabolic Disorders, St Thomas's Hospital Medical School, London SE1
}

\begin{abstract}
Summary
The transport of lipids in plasma, and its dietary regulation are briefly reviewed. Plasma lipid concentrations are low in the neonate, rising to near-adult levels by the age of six years. The causes of childhood hyperlipidaemia are discussed and a classification is proposed.
\end{abstract}

LIPIDs are not carried as such in blood plasma they are virtually insoluble in water. Their presence in plasma is in the form of complex proteins, the lipoproteins (Lewis, 1976); these contain proteins with the ability to solubilize lipids. There are four lipoprotein classes: (1) High density lipoproteins, which contain a large proportion of protein, carry about 1-1.3 mmol/1 of cholesterol, and also phospholipids. (2) The major lipoprotein of human plasma is $\beta$-lipoprotein or low-density lipoprotein. It carries some 3-4 mmol/l, i.e. about two-thirds of the plasma cholesterol and has a greater ratio of lipids to protein than does high density lipoprotein. It also contains a small amount of triglyceride. (3) Pre- $\beta$-lipoprotein, a still large particle, otherwise known as very low density lipoprotein, is the main vehicle of the triglyceride present in plasma during the fasting state, i.e. the endogenous triglyceride. It also carries cholesterol and phospholipids, and the lipid to protein ratio is about $9: 1$. (4) Chylomicrons which appear in plasma during absorption of fat; in alimentary lipaemia they transport most of the dietary fat which has been absorbed. The liver is the main source of very low density lipoprotein; this lipoprotein, and also chylomicrons, are metabolized within the circulation (Fig. 1) (Lewis, 1977). The enzyme lipoprotein lipase hydrolyses most of its triglyceride and some phospholipid, generating a series of smaller and smaller "remnant" particles; within the liver these are converted to low density lipoprotein which is catabolized extra-vascularly in peripheral tissues.

The cholesterol of plasma takes part in a two-way traffic (Fig. 1). The cholesterol secreted in very low density lipoprotein and later borne in low density lipoprotein is largely transported to peripheral tissue, including connective tissue, adipose tissue and other tissue including the smooth muscle of the arterial intima. So the cholesterol contained in the low density lipoprotein (derived from very low density lipoprotein) and chylomicrons, and the triglyceride, are carried from the liver and small bowel and end up in peripheral tissues. By contrast, some of the cholesterol present in high-density lipoprotein is being transported in the opposite direction. High density lipoprotein is synthetized in the liver and released into plasma, but as it traverses the capillary bed of peripheral tissues, it acquires redundant free cholesterol. A major destination of high density lipoprotein cholesterol is the liver, from which it is excreted in the bile. So, high density lipoprotein concentration may well be a determinant of cholesterol mobilization while the concentration of low density lipoprotein and very low density lipoprotein influence cholesterol deposition in peripheral tissues.

Blood lipid concentrations are under genetic, dietary and endocrine control. Only the second of these will now be discussed. A very large number of major nutrients, vitamins, trace elements and energy balance influence plasma lipoprotein levels. Fat intake is the most extensively studied and, on current knowledge, the most important to the epidemiologist concerned with coronary disease. Fats containing saturated long chain fatty acids increase serum cholesterol and triglyceride levels; monounsaturated acids such as oleate have little effect; and polyunsaturated acids such as linoleate decrease cholesterol and triglyceride levels. Cholesterol intake is of uncertain importance. The author's colleague, Mr P. Mistry, has been studying this effect. He finds that a minority of normal subjects show a pronounced rise in serum cholesterol levels when a $750 \mathrm{mg}$ cholesterol supplement is fed for 2-4 weeks; in others the response is smaller or no change is observed. This individual variation is of great interest. Mistry has been studying the regulation of cholesterol synthesis in these subjects, using cultured leucocytes as a model. Hyper-responders differ from hyporesponders in the control of production of the enzyme hydroxymethylglutaryl-coenzyme A (HMGCoA) 

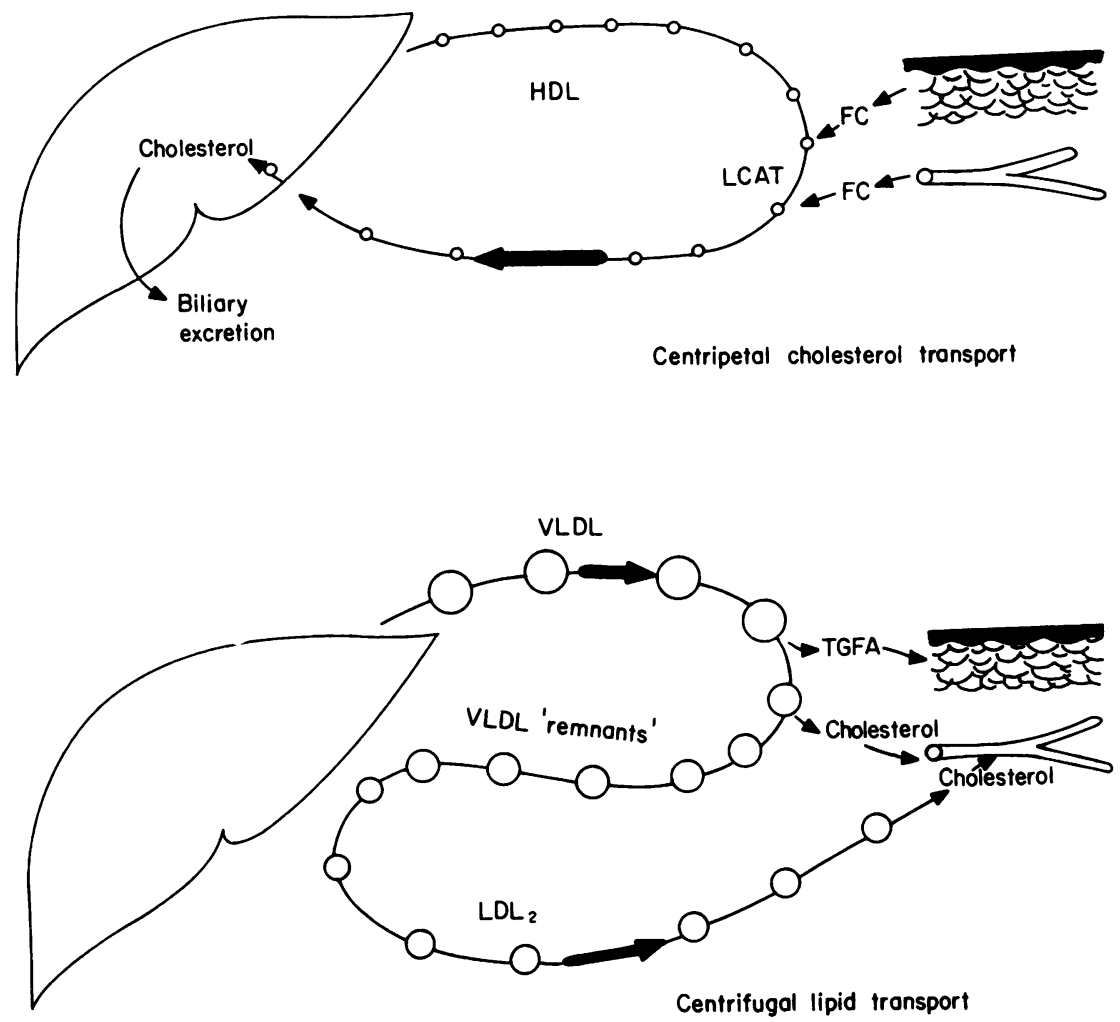

FIG. 1. Plasma cholesterol pathways. HDL $=$ high density lipoprotein; $L D L=$ low density lipoprotein; VLDL = very low density liproprotein; LCAT = lecithin: cholesterol acyltransferase; TGFA = triglyceride fatty acids; FC = free cholesterol.

reductase which regulates cholesterol biosynthesis. No other characteristic has been found which correlates with the response to cholesterol feeding.

Dietary recommendations to reduce serum lipid levels follow the general pattern of decreased saturated fat intake, cholesterol intake and energy intake. Some of the saturated fat is replaced by polyunsaturated and mono-unsaturated fats. There is little consensus as to quantitative advice. In hospital practice one is concerned to maximize the percentage reduction in serum lipids in order to reduce the proportion of hyperlipidaemic patients requiring additional hypolipidaemic drugs. A diet is used containing $35 \%$ of energy from fats, up to $15 \%$ from protein, and less than $300 \mathrm{mg} /$ day of cholesterol. The ratio of polyunsaturated to saturated fats is between $1.5: 1$ and $1.8: 1$, with a fairly high proportion of oleate. This leads to a $15-25 \%$ fall in serum cholesterol and triglyceride levels. There is a major need to define in quantitative terms the optimal diet for population use. When one changes from a saturated to a polyunsaturated fat-containing diet, very low density lipoprotein and low density lipoprotein concentrations fall. This fall may be due to a de:crease in the rate of production of these lipoproteins (Lewis, 1977).

Correction of obesity is an essential step in treat-:ment of most common forms of hyperlipidaemia. $\mathrm{A}^{3}$. steep fall in serum lipid levels precedes the attain-? ment of ideal body weight, and usually persists after the patient has resumed a weight-maintaining diet. A negative calorie balance lowers serum lipid levels adipose tissue mass seems to be a separate importan determinant of cholesterol and triglyceride concen $\frac{\bar{\pi}}{\text {. }}$ trations in plasma.

\section{Changes in lipid concentrations during childhood}

Our knowledge of lipid levels during growth ando adolescence is somewhat incomplete. Most sources indicate that at birth mean serum cholesterol levelsc are about $1.7 \mathrm{mmol} / \mathrm{l}$, with a 95 th percentile of aboute $2.4 \mathrm{mmol} / \mathrm{l}$. Mean triglyceride levels are about $0.4_{0}$ $\mathrm{mmol} / \mathrm{l}$. The cholesterol level in very low, low and5 high density lipoproteins are $0 \cdot 15,0 \cdot 8$ and $0.8 \mathrm{mmol} / \overrightarrow{\mathbb{P}}_{\mathrm{O}}$ 
respectively. Thus high density lipoprotein carries a far higher proportion of circulating cholesterol than in adults; but subsequently its concentration rises only modestly, reaching near-adult levels of about $1 \mathrm{mmol} / \mathrm{l}$ by the age of 1 year. The two-fold rise in serum cholesterol during the first extra-uterine year is largely due to increased levels of low and very low density lipoproteins. These trends continue to the age of 6 years, by which time median levels in Westernized populations are about $4 \mathrm{mmol} / \mathrm{l}$, only a little below those in young adults.

Free fatty acid concentrations are low, about $0.3 \mathrm{mmol} / 1$ in cord blood serum (the fetus is essentially carbohydrate fed); they rise to $1-1.2 \mathrm{mmol}$ in the first $24 \mathrm{hr}$, then decrease to typical adult levels (in the fasted state) of $0.6 \mathrm{mmol} / 1$.

There are major differences in serum cholesterol and triglyceride levels between adult populations, reflecting dietary factors; while there is some disagreement in published data, the balance of information at present suggests that ethnic and geographical differences are slight or absent at birth.

\section{Classifications of hyperlipidaemia}

The obvious first step in investigating a hyperlipidaemic patient is to seek underlying causes, i.e. to recognize secondary hyperlipidaemic states. Of the very many known causes (Lewis, 1976) those of especial importance in paediatric practice are shown in Table 1.

TABLE 1. Secondary hyperlipidaemias in childhood

Glycogen storage disease (Types 1, 3 and 6).

Diabetes.

Idiopathic hypercalcaemia.

Hypothyroidism.

Isolated growth hormone deficiency.

Obstructive jaundice.

In children, as in adults, the majority of hyperlipidaemic patients do not have any demonstrable underlying cause. The hyperlipidaemia is due to various genetic disorders and, even more often, to the interaction of genotype with diet, obesity and other factors (Leading Article, 1977). Their classification is a matter of considerable uncertainty' at the present time. Ten years ago the Fredrickson WHO classification (Fredrickson, Levy and Lees, 1967) became most widely known (Table 2). This depends simply on which lipoprotein class is responsible for the hyperlipidaemia. Another way of classification is based upon the genetic disorders of plasma lipid metabolism, and at least six can be distinguished on current knowledge (Table 3). There are at least two disorders which produce hypercholesterolaemia, both due to a high level of low density lipoprotein in plasma. One is often known as familial hypercholesterolaemia. The disorder is severe, present from birth and confers grave risk of coronary heart disease. It is inherited as a Mendelian dominant in about $3 / 1000$ of the population. A very much commoner cause of hypercholesterolaemia (prevalence figures depend on the definition chosen) is a hypercholesterolaemia which is probably due to multiple genes interacting with other factors such as diet; and it may be that this multifactorial hypercholesterolaemia is a major reason why some communities have higher mean cholesterol levels than others. Another well defined entity is familial combined hyperlipidaemia, in which the affected members of the family may have various forms of hyperlipoproteinaemia causing elevated levels of cholesterol, triglyceride or both lipids. The frequency has been estimated at $2-3 \%$ of the population, and this is the commonest inherited lipoprotein disorder in patients with ischaemic heart disease (Goldstein et al., 1973; Nikkilä and Aro, 1973). Familial hypertriglyceridaemia is seen less frequently. There is a rare entity, sometimes known as Type III or broad beta disease, which appears to be due in part to an accumulation of lipoprotein remnants in plasma. This is inherited as in autosomal dominant but it seems to occur much more commonly in families affected by endogenous hypertriglyceridaemia. Perhaps the rarest of all hyperlipidaemias is due to deficiency of the enzyme lipoprotein lipase and manifests extreme hypertriglyceridaemia with a type I or V lipoprotein pattern. Lastly, a recently described form of familial hyperlipidaemia exists in which mild to moderate hypercholesterolaemia is due to elevated levels of high density lipoprotein. Such patients have no xanthomas or other physical signs suggestive of hyperlipidaemia. They may possess relative immunity to ischaemic heart disease, and often reach an advanced age. The existence of this probably uncommon entity is one justification for obtaining lipoprotein analyses as well as lipid analyses, wherever clinically indicated.

The simplest classification is one which has the pragmatic goal of separating primary hyperlipidaemias into groups differing in their optimal treatment, therapeutic groups 1, 2 and 3 (Table 3) (Lewis, 1976). In group 1 there is hypercholesterolaemia, but normal triglyceride levels; and there are two groups in which hypertriglyceridaemia is present, with or without a rise in serum cholesterol as well. The difference between the common group 2 and the rare group 3 is that in the former the triglyceride is of endogenous origin, and requires treatment chiefly by weight reduction or drugs such as clofibrate; in group 3 there is lipoprotein lipase deficiency producing exogenous hypertriglyceridaemia, treated by a very low fat intake. 
TABLE 2. Fredrickson/WHO classification of primary hyperlipoproteinaemias

\begin{tabular}{llcc}
\hline & \multicolumn{1}{c}{$\begin{array}{c}\text { Abnormality of } \\
\text { lipoprotein }\end{array}$} & Cholesterol & Triglyceride \\
\hline Type I & chylomicrons $\uparrow$ & $\uparrow$ & $\uparrow \uparrow$ \\
Type IIa & LDL $\uparrow$ & $\uparrow \uparrow$ & $\uparrow$ \\
Type IIb & LDL $\uparrow ;$ VLDL $\uparrow$ & $\uparrow$ & $\uparrow$ \\
Type III & cholesterol-rich VLDL $\uparrow$ & $\uparrow \uparrow$ & $\uparrow$ \\
Type IV & VLDL $\uparrow$ & $\mathbf{N} \uparrow$ & $\uparrow \uparrow$ \\
Type V & chylomicrons $\uparrow ;$ VLDL $\uparrow$ & $\uparrow$ & \multicolumn{1}{c}{ V } \\
\hline
\end{tabular}

$\mathrm{LDL}=$ low density lipoprotein; $\mathrm{VLDL}=$ very low density lipoprotein; $\mathrm{N}=$ normal.

TABLe 3. Primary hyperlipoproteincinaemia - classification

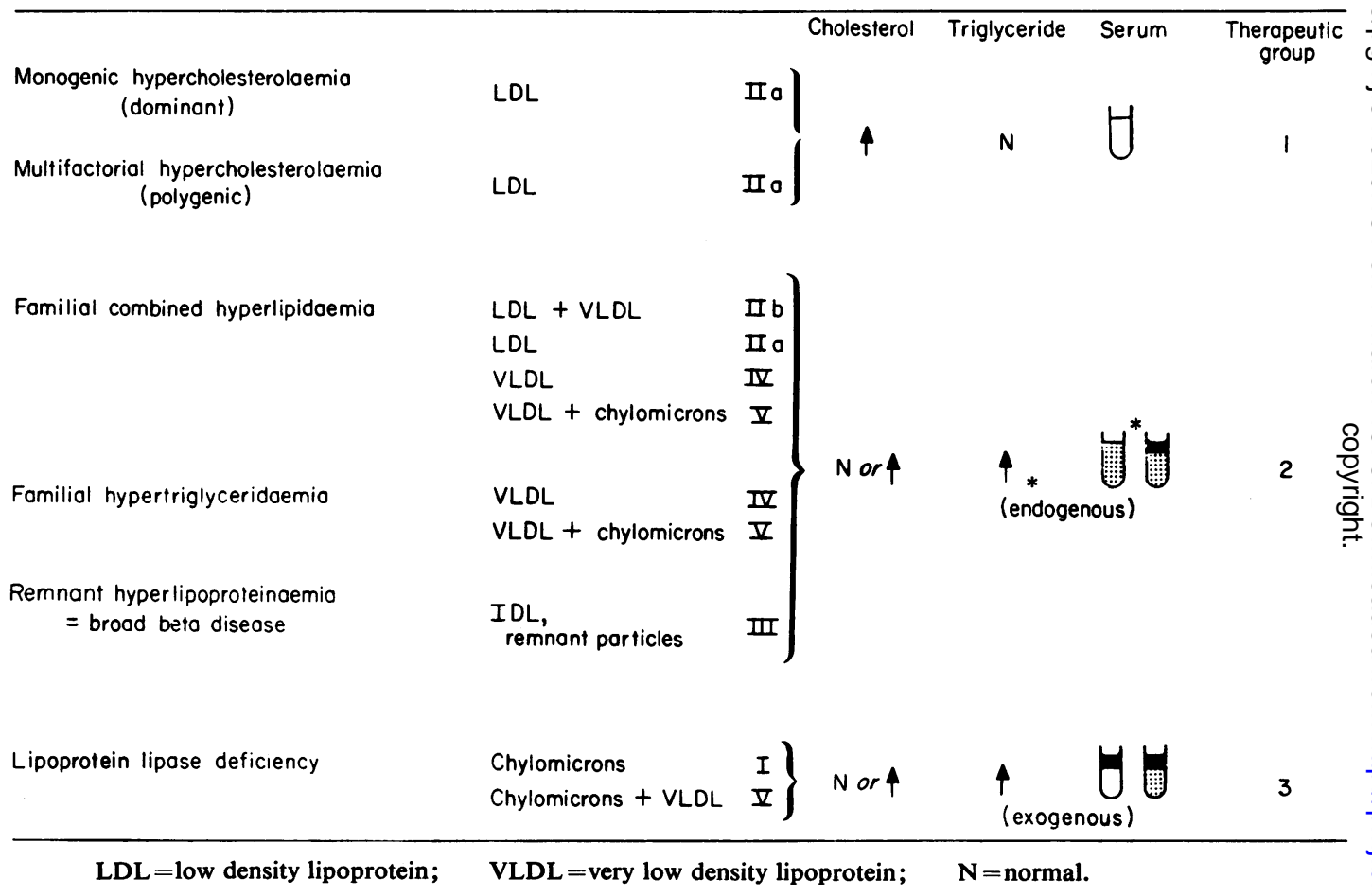

The early treatment of hyperlipidaemia

The author and his colleagues have recently been looking at the relationship between the concentration of plasma low density lipoprotein and the rate at which this lipoprotein enters the arterial intima in man (Niehaus et al., 1977). Overall there is a positive correlation between this rate and the plasma concentration of lipoprotein of about $r=0.5$, but if we separate younger individuals and older ones, then the correlations become very much closer and in young individuals the correlation coefficient is of the order of $r=0.9$, while in older ones it is $r=0 \cdot 7$. Thus low density lipoprotein concentration in plasma is one of the factors determining the rate of lipid deposition in the arterial wall. But clearly cholesterol deposition is a function of not only the lipoprotein concentrations. but also of the duration of the hyperlipidaemia. This perhaps constitutes theo strongest argument for detection and treatment of $\mathrm{F}$ hyperlipidaemia in childhood. There is one ridero that the author would like to make; that is, that many of the inborn disorders of lipid metabolism do not? manifest in children. For example, familial combined hyperlipidaemia is rare until the age of 20 years and ${ }^{\circ}$ endogenous hypertriglyceridaemia (although it has $\sim$ been described even in the neonate) seems to be N much rarer in paediatric practice than in adulto medicine.

\section{References}

Frederickson, D.S., LeVy, R.I. \& LeES, R-S. (1967) Fat $\frac{T}{-}$ transport in lipoproteins: an integrated approach to mechanisms and disorders. New England Journal of Medicine, 276, 32, 94, 148, 215, 273. 
Goldstein, J.L., Schrott, M.G., Hazzard, W.R., Bierman, E.L. \& MotULSKY, A.G. (1973) Hyperlipidemia in coronary heart disease. II. Journal of Clinical Investigation, 52 , 1540.

Leading ARTicle (1977) Diet, energy balance, and genes and serum cholesterol British Medical Journal, 1, 789.

Lew1s, B. (1976) The Hyperlipidaemias: Clinical and Laboratory Practice. Blackwell Scientific Publications, Oxford.
Lewis, B. (1977) Plasma lipoprotein interrelationships. Biochemical Society Transactions, 5, 589.

Niehaus, C.E., Nicoll, A., Wootton, R., Williams, B., Lewis, J., ColtarT, D.J. \& LewIS, B. (1977) Influence of lipid concentrations and age on transfer of plasma lipoprotein into human arterial intima. Lancet, ii, 469.

NikKIL Ä, E.A. \& ARO, A. (1973) Family study of serum lipids and lipoproteins in coronary heart disease. Lancet, $\mathbf{i}$, 954. 